\title{
A Review of Midodrine for the Treatment of Intradialytic Hypotension
}

\author{
Vinay Srinivasa* \\ Toowoomba Hospital, Pechey St, South Toowoomba QLD, Australia
}

*Corresponding author: Vinay Srinivasa, Toowoomba Hospital, Pechey St, South Toowoomba QLD, Australia, E-mail: Vinay.Srinivasa@health. qld.gov.au

Received: 13 Mar, 2019 | Accepted: 22 Apr, 2019 | Published: 26 Apr, 2019

Citation: Srinivasa V (2019) A Review of Midodrine for the treatment of Intradialytic Hypotension. Int J Nephrol Kidney Fail 5(1): dx.doi. org/10.16966/2380-5498.169

Copyright: (c) 2019 Srinivasa V. This is an open-access article distributed under the terms of the Creative Commons Attribution License, which permits unrestricted use, distribution, and reproduction in any medium, provided the original author and source are credited.

\section{Introduction}

Intradialytic hypotension (IDH) is very common and is a source of concern to both health staff and patients. There are several options to treat IDH including prolonging the duration of dialysis sessions, increasing the frequency of sessions and using pharmacological agents [1-3].

Midodrine is one such agent that has been used in the treatment of IDH. It is primarily used in the treatment of orthostatic hypotension secondary to autonomic dysfunction. From 1996 till 2004 small studies were performed, highlighting potential benefit of Midodrine for the treatment of IDH. These studies eventually culminated into a comprehensive meta-analysis performed by Prakash, et al. which evaluated the safety and efficacy of midodrine amongst dialysis patients [4].

Following on from this meta-analysis, no further trials or studies were published assessing the effects of midodrine amongst dialysis patients. In 2010, the US food and drug administration (FDA) proposed to withdraw approval for midodrine citing a lack of evidence and post-marketing proof of efficacy from Shire pharmaceuticals who manufactured the drug [5,6].

This sparked criticism within the medical community, particularly from the nephrology community within the American society of Nephrology (ASN) who issued a public statement opposing the decision made by the FDA. As a result, in 2012, the FDA and Shire pharmaceutical company came to an agreement; the pharmaceutical company would conduct two clinical trials to verify clinical benefit and that Midodrine would remain on the market as off label use [5].

The results of these two trials confirmed that Midodrine was successful for the treatment of orthostatic hypotension. Yet, questions regarding its benefit for the treatment of IDH have remained. Results from the study by Brunelli and group in 2018, have further raised doubt about its benefit in the treatment of IDH [1].

\section{Discussion}

(IDH) is a clinical problem encountered in renal dialysis units and is observed in patients with end stage renal failure (ESRF) on maintenance hemodialysis. It has an incidence rate varying from 10 to $30 \%$ [2,5,7-9]. Significantly, IDH is associated with increased mortality, decreased quality of life, loss of vascular access and myocardial infarction [1-5,8]. Moreover, it can lead to dialysis inadequacy as patients often cut short their time on dialysis due to suffering from symptoms of dizziness, fatigue cramps and blurred vision which can be debilitating $[2,4,8]$.

Defining IDH has been proven to be difficult with various agencies reporting different definitions. The general consensus is though, that it is best defined as a systolic blood pressure of $<100 \mathrm{mmHg}$, or a blood pressure drop of more than $20 \mathrm{mmHg}$ with symptoms $[4,10]$.

The pathogenesis of dialysis hypotension is complex and poorly understood. Originally autonomic dysfunction secondary to uremia was thought to be responsible. It is now believed that transient intravascular hypovolemia is thought to be the major etiological factor behind IDH.

Compensatory physiological mechanisms in response to the induced hypovolemia include alterations in cardiac output by increased heart rate and contractility. Augmentation of cardiac preload by venous capacitance and return, arteriolar venoconstricition and plasma refilling from interstitial fluid and intracellular compartments [11].

There have been several therapeutic measures employed to manage the effects of IDH. These measures include withholding antihypertensive therapy on dialysis days, avoiding large meals on dialysis, sodium profiling, alterations in ultrafiltration rates on dialysis and cooling of the dialysate. As described earlier, increasing the duration and frequency of dialysis sessions has also been performed. Evidence supporting these treatments are however, limited as few prospective trials exist $[1,5,8]$.

Midodrine, an alpha 1 agonist has long been a therapeutic agent for dialysis hypotension, since it was first discovered to be successful in the treatment of orthostatic hypotension in autonomic neuropathy and other forms of orthostatic hypotension such as spinal cord injury [9]. Since 1996, midodrine has been studied to help provide safe and 
relatively symptom free dialysis treatments for patients with ESRF [10,12-14].

Midodrine or Midodrine hydrochloride is the prodrug of the specific alpha 1 adrenergic receptor agonist, desglymidodrine. This metabolite induces constriction of both arterial and venous capacitance vessels, and prevents venous pooling of the blood whilst increasing blood pressure $[12,15]$.

Peak levels of the active metabolite in serum are achieved in 1 hour and its half-life is 3 hours, as measured in individuals with orthostatic and other secondary causes of hypotension $[1,4,5,16]$. Furthermore, it is rapidly absorbed in the gastrointestinal tract, converted to its active metabolite in the systemic circulation and is renally cleared. It is specific for the alpha 1 receptor, and does not cross the blood brain barrier thereby minimizing neurological and cardiovascular effects. Midodrine dosing regimens range from 2.5 to $10 \mathrm{mg}$ orally 15 to 30 minutes prior to the onset of dialysis treatment $[4,16]$.

In published studies from 1996-2004, it was described in the literature that midodrine was reported to be safe effective and well tolerated with minimal side effects in ESRF patients $[4,5,9,17,18]$. Importantly these studies evaluated patients with multiple co-morbidities that included diabetes coronary artery disease and peripheral vascular disease. It should be noted these studies contained a small number of patients with short term follow up. $[4,16]$. Consequently, the long-term effects of midodrine and its outcomes on blood pressure were not established and were largely unknown $[4,5,9,19,12,16]$.

In their case report Rubinstein and group, reported a case of a 48-year-old female on hemodialysis suffering from hypotension. Her background medical history included lupus nephritis and bilateral nephrectomies for renal cell carcinoma. She was commenced on midodrine but 6 months into her treatment developed arterial ischemic ulcers of her toes. Despite being deemed safe, the authors concluded cautionary use of midodrine in patients with peripheral vascular disease. Additional studies have reported multi-systemic adverse effects from midodrine therapy. These include pilomotor reactions; goose bumps, formication, tingling, chills. Gastrointestinal effects: nausea, stomatitis and heartburn. Cardiovascular effects: tachycardia, palpitations, supine hypertension and bradycardia. Central nervous system effects: headache, dizziness, restlessness, excitability, irritability and sleep disturbances. Other reactions that have been described are allergic skin reactions and urinary retention [16]. These adverse reactions have been classified as mild to moderate and it was thought that future trials should investigate the long-term safety profile.

The first description of midodrine being used in dialysis patients was by Blowey in a case report published in 1996. [10]. $5 \mathrm{mg}$ of oral midodrine was administered to an 18-year-old male with BardetBiedel Syndrome on hemodialysis who was experiencing hypotension whilst on dialysis. IDH improved post administration but interestingly it resolved after $10 \mathrm{mg}$ of midodrine. The findings of this report ushered in further studies examining the use of midodrine to treat IDH.

These studies were reviewed by Prakash and group in their excellent systematic review published in 2004. Evidence supporting the use of midodrine for dialysis patients has been cited from this article. Conclusions made from this review affirmed midodrine to be a safe and effective treatment for dialysis induced hypotension. Eleven studies were chosen based on exclusive selection criteria; one study was from unpublished data. Prospective and retrospective observational studies that were pre- and post-intervention in design and crossover studies were included in the analysis. In addition to this, the review required studies that had at least 5 patients on chronic hemodialysis experiencing IDH who were treated with oral midodrine. This requirement was designed to exclude very small case series or case reports. Objective outcomes of interest that were measured included changes in systolic and diastolic blood pressure during dialysis, along with subjective outcomes including adverse effects, intradialytic symptoms including prolonged stay after dialysis or treatment with saline [4].

Of note, the results showed post-dialysis systolic and diastolic blood pressures to be higher during midodrine treatment $v s$ control. Post-dialysis systolic blood pressure was higher by $12.4 \mathrm{mmHg}$ [95\% confidence interval (CI) 7.5-17.7] and diastolic pressure was higher by $7.3 \mathrm{mmHg}$ (95\% CI 3.7-10.9) [4]. Similarly, 6 out of 10 studies described improvement in symptoms of IDH. Importantly no serious adverse events were documented with midodrine. The authors however recognized and acknowledged several limitations of their review, based on the quality of the studies [4].

It was highlighted in their analysis and discussion, that 2 of the 11 studies chosen were crossover in design while the remainder were pre- and post-intervention. There were no published randomized controlled parallel group trials, and the number of patients in each study were small $[4,16]$.

Moreover, it wasn't clear from the studies whether the patients, analyzers or providers were blinded to treatment, thereby exaggerating benefit and causing bias [4]. It was noted, the review did not address the question of whether midodrine offered any added advantage to IDH preventing strategies such as cool dialysate, thermoneutral and or isothermic dialysis [4]

Only two studies in the review compared cool dialysate, midodrine or both with standard therapies. Significantly these studies had a small number of participants. Despite the small number of patients, it was found that there was no discernible difference between cool dialysate and midodrine, nor any added benefit from combination [4].

Similarly, in the studies that measured blood pressure pre-dialysis, those that reported the timing specifically, measured pre-dialysis blood pressures 15 to 30 minutes after the dose of midodrine given. This action may have affected the results of blood pressure readings in both the nadir and post dialysis phase thus overestimating the effects of Midodrine. Baseline blood pressure readings were not equal [4].

The authors identified this as a significant limitation of their analysis, and had suggested to perform a future trial which involves randomized parallel group designs, with blood pressures measured prior to administration of midodrine or placebo. Despite these limitations, the overall findings of the systematic review supported the use of Midodrine for dialysis patients reporting an increase in blood pressure. Furthermore, it was proposed that a larger randomized controlled trial using cool dialysate as the standard of therapy, with which the addition of midodrine or placebo should be compared. It was thought that such a trial would be effective in evaluating the safety profile of midodrine [4].

From 2004 till 2018, no additional studies were performed that examined the effects of Midodrine in dialysis patients. Much of the evidence regarding midodrine use in dialysis patients up to this point in time favored potential benefit. Resultantly, Midodrine has been used as an off-label drug in some dialysis units for the treatment of IDH. The study by Brunelli published in the American Journal of Nephrology in 2018 , is the first study to examine the long-term outcomes of midodrine in dialysis patients. A retrospective study, 1046 patients were prescribed midodrine and were matched to 2037 controls. 
Cardiovascular outcomes were measured. Results of the review showed that patients in the midodrine group had higher mortality rates, all-cause hospitalizations and cardiovascular hospitalizations. (Adjusted incidence rates of 1.37, 1.31 and 1.42 respectively, when compared with controls) [1].

Moreover, users of midodrine during follow up, had lower predialysis systolic blood pressures (SBP), lower nadir SBP, greater fall in SBP during dialysis and experienced more episodes of IDH $[1,15]$. Importantly there are some flaws in the study. It did not disclose the reasons for midodrine administration to patients and medication adherence was not assessed $[1,20]$.

Further limitations include selection bias and residual confounding $[1,20]$. In their editorial, Hamme and group inferred that the increased cardiovascular events were a result of increased blood pressure variability caused by the pharmacological actions of Midodrine. Increased blood pressure variability is well-known to be associated with higher stroke risk and increased cardiovascular events [20-28]. The results of the study have raised doubts on Midodrine improving cardiovascular outcomes amongst hemodialysis patients.

\section{Conclusion}

Dialysis hypotension remains a vexing problem to the nephrologist. It is not easily prevented by pharmacological treatment $[5,16]$. Previous studies had suggested that midodrine could be an effective drug for the treatment of hypotension in dialysis patients. This notion was believed, despite the knowledge, that the studies were of a small sample size and were not randomized $[1,4,20]$. Whilst midodrine seems to be safe in the short-term, the results of the study by Brunelli and group, have cast doubt on midodrine being used long-term for the treatment of IDH $[1,15]$. More studies including randomized controlled trials are required.

\section{References}

1. Brunelli S, Cohen D, Marlowe G, Van Wyck D (2018) The Impact of midodrine on outcomes in patients with intradialytic hypotension. Am J Nephrol 48: 381-388.

2. Rubinstein S, Haimov M, Ross MJ (2008) Midodrine-induced vascular ischemia in a hemodialysis patient: a case report and literature review. Ren Fail 30: 808-812.

3. Sulowicz W, Radziszewski A (2006) Pathogenesis and treatment of dialysis hypotension. Kidney Int 70: S36-S39.

4. Perazella MA (2001) Pharmacologic options available to treat symptomatic intradialytic hypotension. Am J Kidney Dis 38: S26-S36.

5. Chang $T$ (2017) Impact of drugs on intradialytic hypotension: Antihypertensives and vasoconstrictors. Semin Dial 30: 532-536.

6. Dysautonomia International, Action Alert, Keep Midodrine Legal in the US 2012.

7. Blowey D, Balfe JW, Gupta I, Gajaria MM, Koren G (1996) Midodrine efficacy and pharmacokinetics in a patient with recurrent intradialytic hypotension. Am J Kid Dis 28: 132-136.

8. Bradshaw W (2013) Intradialytic hypotension: a literature review. Renal Society of Australasia J 10: 22-29.

9. House $A$ (2011) Are there any contradictions to using midodrine for intradialytic hypotension? Semin Dial 24: 402-403.
10. Alappan R, Cruz D, Abu-Alfa AK, Mahnensmith R, Perazella MA (2001) Treatment of severe intradialytic hypotension with the addition of high dialysate calcium concentration to midodrine and/ or cool dialysate. Am J Kid Dis 37: 294-299.

11. Ronco C (2001) The problem of hypotension in haemodialysis. Nephrol 6: 99-103.

12. Fuujisaki K, Kanai $H$, Hirakata $H$, Nakamura S, Koga $Y$, et al. (2007) Midodrine hydrochloride and I-threo-3, 4 dihydroxy-phenserine Preserve cerebral blood flow in hemodialysis patients with orthostatic hypotension. Ther Apher Dial 11: 49-55.

13. Lin Y, Wang JY, Denq JC, Lin SH (2003) Midodrine improves chronic hypotension in hemodialysis patients. Am J Med Sci 325: 256-260.

14. Montagnac R, Clavel P, Delhotal-Landes B, Flouvat B, Poulain S, et al. (2001) Use of midodrine to treat permanent hypotension in a chronic hemodialysis patient. Clin Nephrol 56: 162-168.

15. Hoeben H, Abu-Alfa AK, Mahnensmith R, Perazella MA (2002) Hemodynamics in patients with intradialytic hypotension treated with cool dialysate or midodrine. Am J Kidney Dis 39: 102-107.

16. Shoji T, Tsubakihara Y, Fujii M, Imai E (2004) Hemodialysis-associated hypotension as an independent risk factor for two-year mortality in hemodialysis patients. Kidney Int 66: 1212-1220.

17. Daugirdas, J (2015) Measuring Intradialytic hypotension to improve quality of care. J Am Soc Nephrol 26: 512-514.

18. Fang J, Huang C (1996) Midodrine hydrochloride in patients on hemodialysis with chronic hypotension. Ren Fail 18: 253-260.

19. Flynn J, Mitchell MC, Caruso FS, McElligott MA (1996) Midodrine treatment for patients with hemodialysis hypotension. Clin Nephrol 45: 261-267.

20. Hammes M, Bakris GL (2018) Intradialytic hypotension-is midodrine the answer? Am J Nephrol, 48: 378-380.

21. Cruz D, Mahnensmith RL, Brickel HM, Perazella MA (1998) Midodrine is effective and safe therapy for intradialytic hypotension- 8 months follow up. Clin Nephrol 50: 101-107.

22. Izhar M (1999) Midodrine in intradialytic hypotension. Int J Artif Organs 22: 529-530.

23. Kitamura M, Saito A (2001) Dialysis hypotension: a review of recent studies of causative factors. Nephrol 6: 109-112.

24. Lim P, Yang CC, Li HP, Lim YT, Yeh CH (1997) Midodrine for the treatment of intradialytic hypotension. Nephron 77: 279-283.

25. McIntyre CW, Salerno FR (2018) Diagnosis and treatment of intradialytic hypotension in maintenance hemodialysis patients. Clin J Am Soc Nephrol 13: 486-489.

26. Palmer BF, Henrich WL (2008) Recent advances in the prevention and management of intradialytic hypotension. J Am Soc Nephrol 19: 8-11.

27. Prakash S, Garg AX, Heidenheim AP, House AA (2004) Midodrine appears to be safe and effective for dialysis-induced hypotension: a systematic review. Nephrol Dial Transplant 19: 2553-2558.

28. Reeves PB, Mc Causland FR (2018) Mechanisms, Clinical Implications, and Treatment of Intradialytic Hypotension. Clin J Am Soc Nephrol 13: 1297-1303. 